governmental organisation and offers free space for social interaction, cognitive and physical stimulation, guidance and support to caregivers.

Objective Describe the sociodemographic, cognitive and affective aspects of the elderly $(n=46)$ and their caregivers $(n=34)$ through a cross-sectional descriptive study evaluating cognitive functioning, dementia, depressive symptoms, performance in basic (BADLs) and instrumental (IADLs) activities of daily living, and emotional overload and common mental disorder of the caregiver.

Results The mean age of the elderly individuals was 77 years-old (SD \pm 9 ), $76 \%$ were women, $67 \%$ live without a partner, $93 \%$ were retired and $50 \%$ had $<4$ years schooling. Average participation in the ADC was 24 months, $79 \%$ presented cognitive impairment and $57 \%$ mild, moderate or severe dementia. For BADLs, $94 \%$ showed some dependence on caregivers, while for IADLs, 100\% were dependent. Among the elderly, $35 \%$ presented depressive symptoms that are correlated with the presence of cognitive impairment $(p=0.004)$. Among the caregivers, $47 \%$ reported emotional overload, while $41 \%$ presented common mental disorders. The relevance of the proposal was verified, considering the fragility of the elderly and the burden and emotional distress of caregivers.

\section{SP1-78 ASTHMA-RELATED HOSPITAL MORBIDITY IN POLAND}

doi:10.1136/jech.2011.142976n.55

${ }^{1} \mathrm{~A}$ Pac, ${ }^{*}{ }^{1} \mathrm{P}$ Gorynski. ${ }^{1}$ Jagiellonian University Medical College, Chair of Epidemiology and Preventive Medicine, Krakow, Poland; ${ }^{2}$ National Institute of Public Health, National Institute of Higiene, Warsaw, Poland

Introduction Asthma prevalence is growing in many countries. The prevalence of asthma is particularly high in developed countries. In Poland asthma morbidity is growing. The aim of this study was to assess the hospital morbidity in Poland and to examine the areabased factors area (socioeconomic, accessibility to the health services and air pollution) that influence morbidity.

Methods We used data from the "Hospitalisation" project in which the National Institute of Health, National Institute of Hygiene collect data on hospital admissions across the country. An asthma hospitalisation was defined as a hospitalisation ICD 10 coded J45 or J46 between 2004 and 2006. Data on the population, healthcare accessibility and the level of air pollution at the voivodship level for the same years was also used.

Results An increase in asthma hospitalisation rates was observed over the study period with regional variation in hospital morbidity. Asthma hospitalisation rates were related to the level of average wage $(\beta=-1.69, p<0.001)$, physician accessibility $(\beta=1.50, p=0.002)$, the percentage of young people in population $(\beta=-0.43, p=0.040)$ and sulphur dioxide air pollution level $(\beta=-0.79 ; p=0.006)$.

Conclusions There is high variation in asthma hospital morbidity according to voivodships in Poland which can be partially explained, at the population level, by access to healthcare services and air pollution.

\section{SP1-79 BIRTH ORDER, GESTATIONAL AGE AND BIRTH WEIGHT AND THE AGE AT ONSET OF PUBERTY: EVIDENCE FROM HONG KONG'S "CHILDREN OF 1997" BIRTH COHORT}

doi:10.1136/jech.2011.142976n.56

L L Hui, ${ }^{*}$ G M Leung, T H Lam, C M Schooling. Life course and Lifestyle Epidemiology Group, School of Public Health, Li Ka Shing Faculty of Medicine, The University of Hong Kong, Hong Kong SAR, China

Introduction Intrauterine and pubertal factors are associated with reproductive and cardiovascular health in both sexes. It is unclear how intrauterine exposures, birth order, gestational age and birth weight, also affect the timing of puberty.
Methods We used interval-censored survival analyses in 3750 boys and 3241 girls (84\% follow-up) in a Chinese birth cohort, "Children of 1997", comprising 88\% of births in Hong Kong in April and May 1997, to examine the adjusted associations of birth order, gestational age and birth weight with age at onset of puberty (Tanner stage II). We also examined whether the associations varied with sex or height at 7 years.

Results Birth order and birth weight were unrelated to the age at onset of puberty, adjusted for sex, gestational age and socioeconomic position. Gestational age had a sex-specific association with age at onset of puberty, and was associated with earlier onset among girls (Time Ratio $0.994,95 \%$ CI 0.991 to 0.997 ) but not boys. None of these associations varied with childhood height.

Conclusion Intrauterine exposures, as proxied by gestational age, birth order and birth weight, had little impact on the timing of the onset of puberty, which was only evident for gestational age among girls. Given that it is unclear whether onset, duration or intensity of puberty is more relevant to the detrimental consequences of early puberty, further studies investigating intrauterine, infant and childhood influences on the duration and intensity of puberty may help unravel the early origins of cardiovascular diseases and breast cancer.

\section{SP1-80 PROGRAM EVALUATION FAMILIAL CANCER GENETIC COUNSELLING IN THE COMMUNITY OF VALENCIA. SPAIN (2005-2010)}

doi:10.1136/jech.2011.142976n.57

1,2M G Sáez, * 1,2D S Trejo. ' Oficina del Plan del Cáncer (Dirección General de Salud Pública), Valencia, Spain; ${ }^{2}$ Centro Superior de Investigación en Salud Pública (CSISP), Valencia, Spain

Background In 2005 the Health Department of the Valencia (Spain), implemented a program of familial cancer. The objective of this program is to reduce cancer incidence and mortality in those with a known genetic predisposition. Through Genetic Counselling Units in Cancer (UCGC) a multidisciplinary team provides information, recommendations and follow-up more appropriate for the patient and family.

Method Descriptive analysis from the start of the program in May 2005 to December 2010, through indicators of organisation and effectiveness: number of people who come to the UCGC, average size of families, rates, appropriate referrals, families with identified mutations, and distribution of the gene results for index cases and family. Results 9.942 people have been treated, 870 was referred from primary care and 9.072 from specialty care. $79 \%$ of the consultations met criteria for genetic studies. The 59\% related to the syndrome of breast and ovarian cancer and $32 \%$ relative to the HNPCC syndrome. Some case index was identified from 3.083 families $(78.7 \%)$ and in $91.3 \%$ of them have done a genetic study. Pathogenic mutations were detected in $21.8 \%$ of these studies. Study is offered to families where pathogenic mutation has been found. The percentage of pathogenic mutations diagnosed in family is approximately $54 \%$.

Conclusions The diagnosis and genetic counselling in cancer, as part of an organised program intended to diagnose early-stage disease or prevent it. To make an assessment of the impact of this program on the health of the population would need to follow the very long term.

\section{SP1-81 AN APPROACH TO COMPARE THE IMPORTANCE OF DIFFERENT CARDIOVASCULAR EXPOSURES AS CONTINUOUS VARIABLES}

doi:10.1136/jech.2011.142976n.58

${ }^{1,2} \mathrm{D}$ Khalili, ${ }^{1} \mathrm{Y}$ Mehrabi, ${ }^{2} \mathrm{~F}$ Hadaegh, ${ }^{2} \mathrm{~N}$ Fahimfar, ${ }^{2} \mathrm{~F}$ Azizi. ${ }^{1}$ School of public health, Shahid Beheshti University of Medical Sciences, Tehran, Iran; ${ }^{2}$ Research 
Institute for Endocrine Sciences, Shahid Beheshti University of Medical Sciences, Tehran, Iran

Introduction Attributable Fraction is the commonest method of describing the proportion of a health outcome attributable to an exposure in an exposed group. It applies to binary variables. Many variables are continuous; changing them to binary variables results in loss of information. Using traditional analyses we compared the importance of cardiovascular disease (CVD) risk factors in a continuous form.

Methods A population based cohort study including 1802 men and 2301 women aged $\geq 40$ years in Tehran. We considered modifiable continuous exposures at baseline and CVD events during 8.5 years of follow-up. Using factor analysis we extracted some uncorrelated and standardised factors, each related to a cluster of continuous variables with the same general feature (eg, systolic and diastolic blood pressure as blood pressure factors or body mass index and waist circumference as anthropometric factor); then, a Cox regression including these factors as scores was conducted to estimate the $\mathrm{RR}$ of the last quintile to the first for each factor. Finally we compared these similar RRs in the model using the Wald test.

Results Anthropometric, blood glucose, blood pressure and cholesterol factors were extracted. The total variance explained by factors was $88.6 \%$ in men and $87.3 \%$ in women. In men all factors had the nearly the same RRs ranging from 1.7 to 2.2 but in women the RR of cholesterol was significantly higher than the others (3.4 vs $1.7-2.5$ ).

Conclusion To prevent CVD, all clusters of risk factors should be considered in control programs. Hypercholesterolaemia maybe more important in women.

\section{SP1-82 COLORECTAL CANCER MORTALITY AND ITS POSSIBLE RELATIONSHIP WITH EXPOSURE TO INDUSTRIAL POLLUTION IN SPAIN}

doi:10.1136/jech.2011.142976n.59

\begin{abstract}
${ }^{1,2} \mathrm{G}$ Lopez-Abente, ${ }^{* 1,2} \mathrm{~J}$ García-Pérez, ${ }^{1,2} \mathrm{P}$ Fernández-Navarro, ${ }^{1,2} \mathrm{E}$ Boldo, ${ }^{1,2} \mathrm{R}$ Ramis. ${ }^{1}$ National Centre for Epidemiology, Carlos III Institute of Health, Madrid, Spain; ${ }^{2}$ CIBER Epidemiología y Salud Pública (CIBERESP), Madrid, Spain
\end{abstract}

The European Pollutant Release and Transfer Register (PRTR) constitute a valuable resource for monitoring health effects of industrial pollution. Our objective is to ascertain whether there might be excess colorectal cancer (CRC) mortality among populations residing in the vicinity of Spanish industrial installations governed by the PRTR.

Methods An ecological study of CRC mortality at a municipal level (8098 towns), over the period 1997-2006. We conducted an exploratory "near vs far" analysis to estimate the RRs of towns at a distance of $<2 \mathrm{~km}$ from installations. The analysis include 24 industrial groups. RRs and their $95 \%$ credible/CI were estimated using Poisson regression models, using two approaches: (A) a conditional autoregressive Bayesian model, with explanatory variables; and (B) a mixed regression model. Integrated nested Laplace approximations were used as a Bayesian inference tool.

Results Statistically significant RR were detected in the vicinity of metal production and processing (RR 1.07; 95\% CI 1.01 to 1.12), mining (RR 1.26; 95\% CI 1.08 to 1.46), paper, pulp and board manufacture (RR 1.07; 95\% CI 1.01 to 1.14), ceramic factories (RR $1.05 ; 95 \%$ CI 1.00 to 1.10 ) and food and beverage production (RR $1.07 ; 95 \%$ CI 1.03 to 1.11 ).

Conclusions Residing in the vicinity of PRTR-registered industries may constitute a risk factor for CRC, since a higher mortality was detected in both men and women residing in towns with such industries nearby. Some of the differences between men and women suggest that there may be a strong, little-studied component of occupational exposure. SP1-83 ALCOHOL CONSUMPTION AND URBANISATION IN NORTH
INDIA: A COMMUNITY SURVEY

doi:10.1136/jech.2011.142976n.60

V Gupta Ritvik, ${ }^{*}$ A Krishnan. All India Institute of Medical Sciences, Delhi, India

Introduction Indian is in a state of epidemiological transition with changes in urbanisation and risk factor profiles. In this context, our objective was to study the epidemiology of alcohol use among urban and rural populations.

Methods The study was conducted in Ballabgarh block, India, during January-October 2006 using the WHO-STEPs approach. Participants were selected using multi-stage sampling for rural and urban stratum (sub-divided across town and slum). We targeted enrolling 250 male and female participants each in 10 year agegroups across 25-65 years and enrolled 5005 participants. In a $20 \%$ sub-sample, information was collected about the exposure to health communication messages regarding alcohol.

Results One rural female reported current alcohol use. The prevalence of current consumption of alcohol, defined as consumption within preceding 12 months, was highest among urban males (26.2\%; 95\% CI 20.8 to 32.1) followed by urban slums $(25.6 \%$; $95 \%$ CI 19.2 to 32.4 ) and rural (23.2\%; 95\% CI 18.2 to 28.9). Among alcohol consumers $15.3 \%$ urban, $7.3 \%$ slum and $15.3 \%$ rural males reported reduction in alcohol use in preceding 1 year. $7.2 \%$ urban $10.9 \%$ slum and $7.2 \%$ rural respondents who were current alcohol users reported intermittent cessation of alcohol consumption in preceding 1 year. $59.2 \%(n=603)$ urban, $27.2 \%(n=185)$ urban-slum and $39.5 \%(n=646)$ rural respondents reported receiving information (IEC) on health effects of alcohol with Mass media as the most common source of information.

Conclusion Alcohol is a public health problem among males in Ballabgarh area. Interventions should focus on alcohol cessation services and improved IEC. Urbanisation does not appear to be associated with alcohol use in this community.

\section{SP1-84 DIFFERENT METHODS TO CALCULATE POPULATION ATTRIBUTABLE FRACTION OF RISK FACTORS FOR CARDIOVASCULAR DISEASES: TEHRAN LIPID AND GLUCOSE STUDY (TLGS)}

doi:10.1136/jech.2011.142976n.61

S S Azimi, ${ }^{*}$ D khalili, F Hadaegh, P Yavari. Prevention of Metabolic Disorders Research Center, Tehran, Iran

Background The population attributable fraction (PAF) that estimates potentially community-level effect of risk factors can be useful in planning public health interventions. This study compared different methods for calculating adjusted PAFs for cardiovascular diseases (CVD) in a cohort study with 10 years of follow-up.

Methods Baseline data were employed from 6630 participants (3746 women) above 30 years old and 558 CVD events (238 women) detected during follow-up. Unadjusted approach using Levin's formula, Miettinen formula approach using adjusted OR and HR estimated from logistic and Cox regression and direct estimation of average PAF from logistic regression using Rückinger method, were applied.

Result Estimated PAFs, using HR comparing OR, in both Levin's and Miettinen's formula, with tiny decrease, gave very similar results. However, according to the average PAF method, frankly, we reach to lower fractions; highest modifiable cardiovascular risk factor PAFs, in sequence, was hypertension $(16.2 \%)$, smoking $(14.8 \%)$, diabetes $(10.1 \%)$, hypercholesterolaemia (8.5\%) for men, and hypertension (25.6\%), diabetes (18\%), hypercholesterolaemia $(10.7 \%)$, for women. Also PAF of Age $\geq 60$ years and premature family history of CVD, as most important non-modifiable CVD risk 